Georgian Mathematical Journal

Volume 13 (2006), Number 1, 183-191

\title{
WEIGHTED INEQUALITIES FOR INTEGRAL OPERATORS WITH ALMOST HOMOGENEOUS KERNELS
}

\author{
MARTA URCIUOLO
}

\begin{abstract}
Let $m \in N$ and $a_{1}, \ldots, a_{m}$ be real numbers such that for each $i$, $a_{i} \neq 0$ and $a_{i} \neq a_{j}$ if $i \neq j$. In this paper we study integral operators of the form

$$
T f(x)=\int k_{1}\left(x-a_{1} y\right) \cdots k_{m}\left(x-a_{m} y\right) f(y) d y,
$$

with $f, \varphi_{i, j}: R^{n} \rightarrow R, k_{i}(y)=\sum_{j \in Z} 2^{\frac{j n}{q_{i}}} \varphi_{i, j}\left(2^{j} y\right), 1 \leq q_{i}<\infty, i=1, \ldots, m$, $\frac{1}{q_{1}}+\cdots+\frac{1}{q_{m}}=1$.

If $\varphi_{i, j}$ satisfy certain uniform regularity conditions out of the origin, we obtain the boundedness of $T: L^{p}(w) \rightarrow L^{p}(w)$ for all power weights $w$ in adequate Muckenhoupt classes.
\end{abstract}

2000 Mathematics Subject Classification: 42B25, 42A50, 42B20.

Key words and phrases: Weights, integral operators.

\section{INTRODUCTION}

In [7] the authors obtain the $L^{p}$ boundedness, $p>1$, for a class of maximal operators on the three-dimensional Heisenberg group. The operators they consider have relevance in the analysis on $S l\left(R^{3}\right)$. Some of them actually arise in the study of the boundary behavior of Poisson integrals on the symmetric space $S l\left(R^{3}\right) / S O(3)$. To obtain the principal results, they analyze the $L^{2}(R)$ boundedness of singular integral operators of the form

$$
T f(x)=\int|x-y|^{-\alpha}|x+y|^{\alpha-1} f(y) d y
$$

$0<\alpha<1$.

A natural question was if these operators were also bounded on $L^{p}(R)$ for $p>1, p \neq 2$ and if this result still holds for larger dimensions or for more general kernels. In [4] we study integral operators of the form

$$
T f(x)=\int_{R^{n}}|x-y|^{-\alpha}|x+y|^{-n+\alpha} f(y) d y,
$$

$0<\alpha<n$. We obtain the $L^{p}\left(R^{n}, d x\right)$ boundedness, $1<p<\infty$, and the weak type $(1,1)$ of them. We observe that the kernel is homogeneous of degree $-n$.

We take the Hardy-Littlewood maximal function as

$$
M f(x)=\sup _{Q} \frac{1}{|Q|} \int_{Q}|f(x)| d x
$$


where the supremum is taken along all the cubes $Q$ such that $x$ belongs to $Q$. We recall that a weight $w$ is a measurable, nonnegative and locally integrable function. It is well known that, for $p>1, M$ is bounded on $L^{p}(w)$ if and only if there exists $c>0$ such that

$$
\sup _{Q}\left(\frac{1}{|Q|} \int_{Q} w\right)\left(\frac{1}{|Q|} \int_{Q} w^{-\frac{1}{p-1}}\right)^{p-1} \leq c .
$$

The class of functions that satisfy this inequality is called $A_{p}$. For $p=1$, the class $A_{1}$ is defined by

$$
M w(x) \leq c w(x)
$$

a.e. $x \in R^{n}$ and for some positive constant $c>0$. The weak type $1-1$ of a maximal function is equivalent to $w \in A_{1}$. These classes $A_{p}$ have been defined by Muckenhoupt (see [6]) in the one-dimensional case and for larger dimensions by Coifmann and Fefferman (see [1]).

In $[9]$ the author proves very general weighted norm inequalities for maximal operators of the form

$$
M_{\mu} f(x)=\sup _{j}\left|f * \mu_{j}(x)\right|,
$$

where $\left\{\mu_{j}\right\}_{j \in Z}$ is a family of finite Borel measures on $R^{n}$, each one supported in $\|x\| \leq 2^{j}$, satisfying a certain decay of its Fourier transform. He also considers the singular integral operator $T_{\sigma} f=\sum_{j=-\infty}^{\infty} \sigma_{j} * f$, for a sequence $\left\{\sigma_{j}\right\}_{j \in Z}$ of signed measures on $R^{n}$ with $\int d \sigma_{j}=0$ that fall under the general theorems of J. Duoandikoetxea and J. L. Rubio de Francia stated in [3], which is an excellent reference for the unweighted theory. In particular the author studies the weight theory for

$$
T_{\Omega} f(x)=p . v \int f(x-y) \frac{\Omega(y)}{|y|^{n}} d y,
$$

where $\Omega$ is homogeneous of degree 0 and has mean value zero on $S^{n-1}$.

Let $m \in N$, let $a_{1}, \ldots, a_{m}$ be real numbers such that for each $i, a_{i} \neq 0$ and $a_{i} \neq a_{j}$ if $i \neq j$. Let $q_{1}, \ldots, q_{m}$ be real numbers, $1 \leq q_{i}<\infty$, such that

$$
\frac{1}{q_{1}}+\cdots+\frac{1}{q_{m}}=1
$$

For each $1 \leq i \leq m$ let $\left\{\varphi_{i, j}\right\}_{j \in Z}$ be a family of nonnegative real functions defined on $R^{n}$, satisfying

H1) $\operatorname{supp} \varphi_{i, j} \subset\left\{y \in R^{n}: 2^{-1} \leq|y| \leq 2\right\}$.

H2) For each $1 \leq i \leq m$ there exists $p_{i}>q_{i}$ such that $\left\|\varphi_{i, j}\right\|_{p_{i}} \leq c$, with $c$ independent of $j$.

Let $k_{i}(x)=\sum_{j \in Z} 2^{\frac{j n}{q_{i}}} \varphi_{i, j}\left(2^{j} x\right)$ and let $T$ be the integral operator with kernel

$$
k(x, y)=k_{1}\left(x-a_{1} y\right) \cdots k_{m}\left(x-a_{m} y\right)
$$


so that for a measurable and nonnegative $f$,

$$
T f(x)=\int k(x, y) f(y) d y .
$$

In [5] we prove that $T$ extends to a bounded operator on $L^{p}\left(R^{n}\right)$. We observe that if $\varphi_{i, j} \equiv \varphi_{i, j^{\prime}}$ for all $j$, then $j^{\prime} \in Z, k_{i}\left(2^{l} x\right)=2^{-l \frac{n}{q_{i}}} k_{i}(x)$ for $l \in Z$, so it is "homogeneous" of degree $-\frac{n}{q_{i}}$ and then $k$ is "homogeneous" of degree $-n$. Many authors have studied singular integral operators with kernels of the form $\sum_{j=-\infty}^{\infty} 2^{-j n} k\left(2^{-j x}\right)$, where $k \in L^{1}\left(R^{n}\right)$, having with some cancellation property and whose Fourier transform has a reasonable decay at infinity (see Theorem 8.23 in [2]). Such kernels have just one singularity at the origin. Now, our kernels have $m$ different singularities.

In [8] we study the case $k_{i}(x)=|x|^{-\alpha_{i}}, \alpha_{1}+\cdots+\alpha_{m}=n$ and obtain weighted inequalities for a wide class of weights $w$ in $A_{p}$.

We recall that a power weight of the form $|x|^{a}$ belongs to $A_{p}$ if and only if $-n<a<n(p-1)$ (see [2]). In this paper we prove

Theorem. Let the operator $T$ be defined by (1). Let $p_{m+1}$ be defined by $\frac{1}{p_{1}}+\cdots+\frac{1}{p_{m}}+\frac{1}{p_{m+1}}=1$ and let $w(x)=|x|^{a}$ with $-n<a<n\left(\frac{p}{p_{m+1}}-1\right)$. If $\max _{1 \leq i \leq m}\left\{\frac{p_{i}}{p_{i}-q_{i}}\right\}<p<\infty$, then $T$ is a bounded operator on $L^{p}(w)$.

We also give some example of kernels of the above described type, for which the condition $-n<a<n\left(\frac{p}{p_{m+1}}-1\right)$ becomes necessary for the boundedness, on $L^{p}\left(|x|^{a} d x\right)$, of the corresponding operator.

Throughout this paper, $c$ will denote a positive constant not necessarily the same at each occurrence.

\section{Proof of the TheOrem}

We will prove that for all $\max _{1 \leq i \leq m}\left\{\frac{p_{i}}{p_{i}-q_{i}}\right\}<p<\infty$, we obtain the weak type condition

$$
w\{x:|T f(x)|>\lambda\} \leq \frac{c}{\lambda^{p}}\|f\|_{p, w}^{p}
$$

and the theorem will follow from the Marcinkiewicz interpolation theorem.

For $x \in R^{n}-\{0\}$ we define $l=l(x) \in Z$ such that $2^{l}<|x| \leq 2^{l+1}$. We take positive numbers $d<1, D>1$ such that

$$
d<\min \left(\min _{1 \leq i \leq m}\left\{\frac{\left|a_{i}\right|}{2},\right\}, \min _{i \neq s}\left\{\frac{\left|a_{i}-a_{s}\right|}{2},\right\}\right)
$$

and $D>2 \max _{1 \leq i \leq n}\left\{\left|a_{i}\right|\right\}$. We define $r<0$ and $R>0$ such that $2^{r}<d \leq 2^{r+1}$ and $2^{R}<D \leq 2^{R+1}$, and we set

$$
A_{i}=A_{i}(x)=\left\{y \in R^{n}:\left|y-a_{i} x\right| \leq 2^{l} d\right\}, \quad 1 \leq i \leq m,
$$




$$
A_{m+1}=\left\{y \in R^{n}:|y| \leq 2^{l} D\right\} \bigcap\left(\bigcup_{1 \leq i \leq m} A_{i}\right)^{c}
$$

and

$$
A_{m+2}=\left\{y \in R^{n}:|y|>2^{l} D\right\} \bigcap\left(\bigcup_{1 \leq i \leq m+1} A_{i}\right)^{c} .
$$

We also define, for $1 \leq i \leq m+2$, operators $T_{i}$ by

$$
T_{i} f(x)=\int_{A_{i}(x)} k(x, y)|f(y)| d y .
$$

In order to obtain (1), we decompose

$$
\{x:|T f(x)|>\lambda\}=\bigcup_{i=1}^{m+2}\left\{x: T_{i} f(x)>\lambda /(m+2)\right\},
$$

and measure each one of these sets separately.

If $2^{l} \leq|x|<2^{l+1}, y \in A_{i}(x)$ and $2^{j}\left(y-a_{i} x\right) \in \operatorname{supp} \varphi_{i, j}$, then

$$
2^{-1} \leq 2^{j}\left|y-a_{i} x\right|<2^{j+l+r+1}
$$

and so $j \geq-l-r-2$. Also, for $s \neq i$,

$$
\left|y-a_{s} x\right| \leq\left|y-a_{i} x\right|+\left|\left(a_{i}-a_{s}\right) x\right| \leq(d+D) 2^{l}
$$

and

$$
\left|y-a_{s} x\right| \geq\left|\left(a_{i}-a_{s}\right) x\right|-\left|y-a_{i} x\right| \geq d 2^{l},
$$

and so, if $2^{k}\left|y-a_{s} x\right| \in \operatorname{supp} \varphi_{s, k}$, then $2^{-1} \leq 2^{k}\left|y-a_{s} x\right| \leq(d+D) 2^{k+l}$ and $2 \geq 2^{k}\left|y-a_{s} x\right| \geq d 2^{k+l}$, then $-l-R-3 \leq k \leq-l-r+1$. So

$$
\begin{aligned}
\int_{A_{i}(x)}|k(x, y) f(y)| d y \leq & c \int_{A_{i}(x)} \sum_{j \geq-l-r-2} 2^{\frac{n j}{q_{i}}}\left|\varphi_{i, j}\left(2^{j}\left(y-a_{i} x\right)\right)\right| \\
& \times \prod_{s \neq i} 2^{\frac{-n l}{q_{s}}}\left|\varphi_{s,-l}\left(2^{-l}\left(y-a_{s} x\right)\right) f(y)\right| d y \\
\leq & c \sum_{\substack{h \geq 0, j \geq-l-r-2}} \int_{2^{l-h-1} \leq\left|y-a_{i} x\right| \leq d 2^{l-h}} 2^{\frac{n j}{q_{i}}}\left|\varphi_{i, j}\left(2^{j}\left(y-a_{i} x\right)\right)\right| \\
& \times \prod_{s \neq i} 2^{\frac{-n l}{q_{s}}}\left|\varphi_{s,-l}\left(2^{-l}\left(y-a_{s} x\right)\right) f(y)\right| d y,
\end{aligned}
$$

but if $2^{j}\left(y-a_{i} x\right) \in \operatorname{supp} \varphi_{i, j}$ and $2^{l-h-1} \leq\left|y-a_{i} x\right| \leq 2^{l-h}$, then $2^{-1} \leq$ $2^{j}\left|y-a_{i} x\right| \leq 2^{l-h+j}$ and $2^{l-h-1+j} \leq 2^{j}\left|y-a_{i} x\right| \leq 2$, so $h-l-1 \leq j \leq h-l+2$. Again, for the sake of simplicity, we study only the case $j=h-l$, since the other cases are similar.

Then

$$
\int_{A_{i}(x)}|k(x, y) f(y)| d y
$$




$$
\begin{aligned}
& \leq c \sum_{h \geq 0} \int_{2^{l-h-1} \leq\left|y-a_{i} x\right| \leq 2^{l-h}} 2^{n \frac{h}{q_{i}}-l}\left|\varphi_{i, h-l}\left(2^{h-l}\left(y-a_{i} x\right)\right)\right| \\
& \times \prod_{s \neq i}\left|\varphi_{s,-l}\left(2^{-l}\left(y-a_{s} x\right)\right) f(y)\right| d y
\end{aligned}
$$

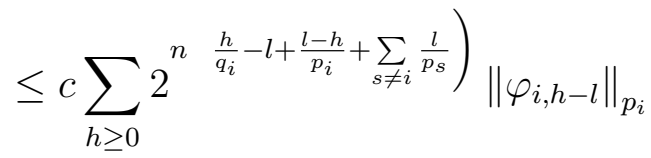

$$
\begin{aligned}
& \times \prod_{s \neq i}\left\|\varphi_{s,-l}\right\|_{p_{s}}\left\|f \chi_{2^{l-h-1} \leq\left|y-a_{i} x\right| \leq 2^{l-h}}\right\|_{p_{m+1}}
\end{aligned}
$$

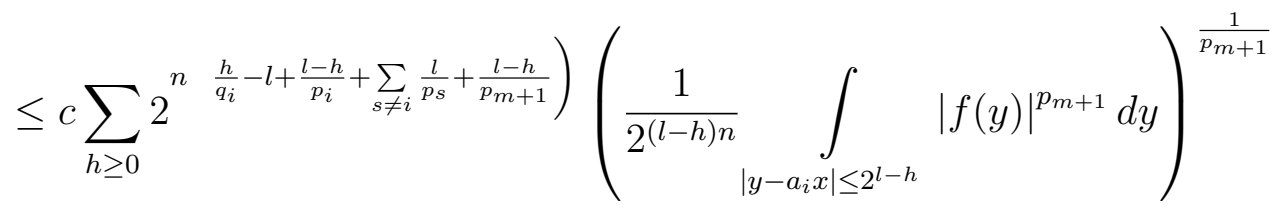

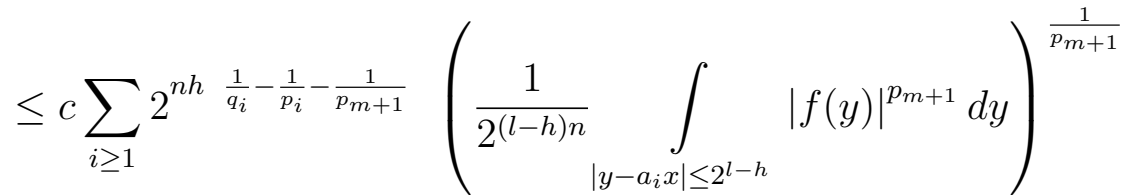

$$
\begin{aligned}
& \leq c\left(M\left(f^{p_{m+1}}\left(a_{i} x\right)\right)\right)^{\frac{1}{p_{m+1}}} .
\end{aligned}
$$

The second inequality follows by Hölder's inequality and the last one follows since $p_{s}>q_{s}, 1 \leq s \leq m$ and so $\frac{1}{q_{i}}-\frac{1}{p_{i}}-\frac{1}{p_{m+1}}=\frac{1}{q_{i}}-1+\sum_{s \neq i} \frac{1}{p_{s}}<\frac{1}{q_{i}}-1+\sum_{s \neq i} \frac{1}{q_{s}}<0$.

Thus

$$
\begin{aligned}
w\left\{x: \int_{A_{1}(x)}|k(x, y) f(y)| d y>\lambda\right\} & \leq w\left\{x: M\left(f^{p_{m+1}}\left(a_{i} x\right)\right)>c \lambda^{p_{m+1}}\right\} \\
& \leq c \frac{1}{\left(\lambda^{p_{m+1}}\right)^{\frac{p}{p_{m+1}}}}\left\|f^{p_{m+1}}\right\|_{\frac{p}{p_{m+1}}}^{\frac{p}{p_{m+1}}}=\frac{c}{\lambda^{p}}\|f\|_{p, w}^{p} .
\end{aligned}
$$

Now, it is easy to check that $p>\max _{1 \leq i \leq m}\left\{\frac{p_{i}}{p_{i}-q_{i}}\right\}$ implies $p>p_{m+1}$. Indeed,

$$
\begin{aligned}
\frac{1}{p_{m+1}} & =1-\left(\frac{1}{p_{1}}+\cdots+\frac{1}{p_{m}}\right)=\left(\frac{1}{q_{1}}+\cdots+\frac{1}{q_{m}}\right)-\left(\frac{1}{p_{1}}+\cdots+\frac{1}{p_{m}}\right) \\
& =\frac{p_{1}-q_{1}}{q_{1} p_{1}}+\cdots+\frac{p_{m}-q_{m}}{q_{m} p_{m}}=\frac{1}{q_{1}}\left(\frac{p_{1}-q_{1}}{p_{1}}\right)+\cdots+\frac{1}{q_{m}}\left(\frac{p_{m}-q_{m}}{p_{m}}\right) \\
& \geq \min _{1 \leq i \leq m}\left\{\frac{p_{i}-q_{i}}{p_{i}}\right\}>\frac{1}{p},
\end{aligned}
$$

then the last inequality follows since $w \in A_{\frac{p}{p_{m+1}}}$ and so $M$ is of weak type $\frac{p}{p_{m+1}}$ with respect to the weight $w$. 
Now, for $y \in\left(\bigcup_{1 \leq i \leq m} A_{i}\right)^{c}$,

$$
\left|y-a_{i} x\right| \leq\left|y-a_{s} x\right|+\left|\left(a_{s}-a_{i}\right) x\right| \leq\left(1+\frac{D}{d}\right)\left|y-a_{s} x\right| .
$$

If $2^{j}\left(y-a_{i} x\right) \in \operatorname{supp} \varphi_{i, j}$ and $2^{k}\left(y-a_{s} x\right) \in \operatorname{supp} \varphi_{s, k}$, we obtain as before that $j, k \leq-l-r+1$. We also have

$$
\frac{1}{2} \leq 2^{j}\left|y-a_{i} x\right| \leq 2^{j}\left(1+\frac{D}{d}\right)\left|y-a_{s} x\right| \leq 2^{j-k} 2\left(1+\frac{D}{d}\right) \leq 2^{j-k+R-r+2},
$$

so $j>k-R+r-3$ and, analogously,

$$
2^{j-k} \frac{1}{2\left(1+\frac{D}{d}\right)} \leq 2^{j-k} \frac{1}{\left(1+\frac{D}{d}\right)} 2^{k}\left|y-a_{s} x\right| \leq 2^{j}\left|y-a_{i} x\right| \leq 2,
$$

so $j<k+R-r+3$. Again we estimate only the case $j=k$.

For $y \in A_{m+1}$, we also have $\left|y-a_{i} x\right| \leq 2^{l-1} \frac{D}{3}$ and so if $2^{j}\left|y-a_{i} x\right| \in$ $\operatorname{supp} \varphi_{i, j}$, then $-l-R-1 \leq j \leq-l-r+1$. Thus we obtain

$$
\begin{gathered}
\int_{A_{m+1}}|k(x, y) f(y)| d y \leq c \int_{\left\{y:|y| \leq 2^{l} D\right\}} 2^{-n l} \prod_{i=1}^{m}\left|\varphi_{i,-l}\left(2^{-l}\left(y-a_{i} x\right)\right)\right||f(y)| d y \\
\leq c 2^{-n l+\sum_{i=1}^{m} 2^{\frac{n l}{p_{i}}}}\left(\int_{\{y:|y| \leq D|x|\}}|f(y)|^{p_{m+1}}\right)^{\frac{1}{p_{m+1}}}=\left(2^{-n l} \int_{\{y:|y| \leq D|x|\}}|f(y)|^{p_{m+1}}\right)^{\frac{1}{p_{m+1}}} \\
\leq c\left(M f^{p_{m+1}}(x)\right)^{\frac{1}{p_{m+1}}},
\end{gathered}
$$

and proceed as in the first case to obtain the desired result.

Finally, by Hölder's inequality

$$
\int_{A_{m+2}}|k(x, y) f(y)| d y \leq\left\|k(x, .) \chi_{A_{m+2}} w^{-\frac{1}{p}}\right\|_{p}\left\|f \chi_{A_{m+2}} w^{\frac{1}{p}}\right\|_{p},
$$

now, Minkowsky's integral inequality implies that

$$
\begin{aligned}
\left\|k(x, .) \chi_{A_{m+2}} w^{-\frac{1}{p}}\right\|_{p} & \leq c \sum_{j \leq-l-r-1} 2^{j n}\left(\int_{A_{m+2}} \prod_{i=1}^{m} \varphi_{i, j}^{p^{\prime}}\left(2^{j}\left(y-a_{i} x\right)\right)|y|^{-\frac{a p^{\prime}}{p}} d y\right)^{\frac{1}{p^{\prime}}} \\
& \leq c \sum_{j \leq-l} 2^{j n+j \frac{a}{p}-\sum_{i=1}^{m} \frac{j n}{p_{i}}} \prod_{i=1}^{m}\left\|\varphi_{i, j}\right\|_{p^{\prime} q_{i}} \leq c|x|^{\frac{-a}{p}+\frac{n}{r_{2}}} .
\end{aligned}
$$

The second inequality follows since the hypothesis $p>\max _{1 \leq i \leq m}\left\{\frac{p_{i}}{p_{i}-q_{i}}\right\}$ implies $p^{\prime} q_{i}<p_{i}, 1 \leq i \leq m$, and since, for the involved $i, j^{\prime} s,|y| \leq\left|y-a_{i} x\right|+\left|a_{i} x\right| \leq$ $2^{-j+1}+D 2^{l+1} \leq c 2^{-j}$. 
From this last inequality we obtain

$$
\int_{A_{m+2}}|k(x, y) f(y)| d y \leq c|x|^{\frac{-a}{p}-\frac{n}{p}}\|f\|_{p, w},
$$

and so

$$
\begin{aligned}
& w\left\{x: \int_{A_{m+2}}|k(x, y) f(y)| d y>\lambda\right\} \leq w\left\{x: c|x|^{\frac{-a}{p}-\frac{n}{p}}\|f\|_{p, w}>\lambda\right\} \\
& \leq w\left\{x:|x|<\left(\frac{\|f\|_{p, w}}{c \lambda}\right)^{\frac{p}{n+a}}\right\}=\int_{|x|<{\frac{\|f\|_{p, w}}{c \lambda}}^{\frac{p}{n+a}}|y|^{a} d y=c\left(\frac{\|f\|_{p, w}}{\lambda}\right)^{p} .}
\end{aligned}
$$

\section{Necessary Conditions}

Next, we give some examples of operators $T$ defined by (1) for which the condition $-n<a<n\left(\frac{p}{p_{m+1}}-1\right)$ becomes necessary for the boundedness of $T$ on $L^{p}\left(|x|^{a} d x\right), 1 \leq p<\infty$.

Example 1. $k_{i}(x)=|x|^{-\alpha_{i}}, \alpha_{1}+\cdots+\alpha_{m}=n$. It can be seen as $\sum_{j \in Z} 2^{j \alpha_{i}} \frac{\chi_{B_{1}(x)}}{|x|^{\alpha_{i}}}$ with $B_{1}=\left\{x \in R^{n}: \frac{1}{2}<|x| \leq 1\right\}$. So $\varphi_{i, j}(x)=\varphi_{i}(x)=\frac{\chi_{B_{1}}(x)}{|x|^{\alpha_{i}}}, q_{i}=\frac{n}{\alpha_{i}}, 1 \leq i \leq$ $m$. Since any $\varphi_{i}$ belongs to $L^{\infty}\left(B_{1}\right)$, it belongs to $L^{p_{i}}$ for all $1 \leq p_{i} \leq \infty$, too. So we will show that if the operator $T$ defined by (1) is bounded on $L^{p}\left(|x|^{a} d x\right)$ then $-n<a<n(p-1)$. It is a reciprocal result of the theorem proved in [8], at least for power weights in $A_{p}$.

Let us prove this assertion. $a>-n$ is necessary for the local integrability of $|x|^{a}$. To check the other inequality, we take $B_{l}=\left\{x \in R^{n}: 2^{-l}<|x| \leq 2^{-l+1}\right\}$, $f=\chi_{B_{1}}$ and $l \gg 1$.

$$
\begin{aligned}
\|T f\|_{L^{p}\left(|x|^{a} d x\right)}^{p} & \geq \int\left|\int_{B_{1}} \prod_{1 \leq i \leq m}\right| y-\left.\left.a_{i} x\right|^{-\alpha_{i}} d y\right|^{p}|x|^{a} d x \\
& \geq \int_{B_{l}}\left|\int_{B_{1}}\right| y-\left.\left.a_{i} x\right|^{-\alpha_{i}} d y\right|^{p}|x|^{a} d x \geq \int_{B_{l}}|x|^{-n p+a} d x \geq 2^{l(a+n(1-p))} .
\end{aligned}
$$

Since $2^{l(a+n(1-p))}$ must be bounded for $l \gg 1$, we obtain $a+n(1-p)<0$.

Example 2. We take $n=1, a_{1}=1, a_{2}=-1, q_{1}=q>q^{\prime}=q_{2} \cdot \varphi_{1}(x)=$ $\frac{\chi_{[1 / 2,1]}(x)}{|x-1 / 2|^{1 / 2 q}}, \varphi_{2}(x)=\frac{\chi_{[1 / 2,1]}(x)}{|x|^{1 / q}}$ and $\varphi_{1, j}(x)=\varphi_{1}(x), \varphi_{2, j}(x)=\varphi_{2}(x), j \in Z$. We observe that $k_{2}(x)=\frac{1}{|x|^{1 / q}}$ a.e. It is easy o check that $\varphi_{i, j}, i=1,2$, satisfy the hypotheses H1) and H2) stated in the introduction for any $p_{1}$ and $p_{2}$ such that $q \leq p_{1}<2 q, q^{\prime} \leq p_{2}<\infty$. Let $p_{3}$ be defined by $\frac{1}{p_{3}}+\frac{1}{2 q}=1$. We suppose 
that the operator $T$ defined by (1) is bounded on $L^{p}\left(|x|^{a} d x\right)$, for some $p$ with $1 \leq p<\infty$. We will show that $-1<a<\frac{p}{p_{3}}-1$.

Indeed, $a>-1$ is necessary for the local integrability of $|x|^{a}$. To obtain the other inequality, we take $j \ll 0$ and set $I_{j}=\left(2^{j-1,2^{j}}\right]$ and $f=\chi_{I_{1}}$.

$$
\begin{aligned}
T f(x) & \geq \int_{I_{1}} 2^{j / q} \varphi_{1}\left(2^{j}(y-x)\right) k_{2}((y+x)) d y \\
\|T f\|_{L^{p}\left(|x|^{a} d x\right)}^{p} & \geq \int_{I_{-j-2}} 2^{j p / q}\left|\int_{I_{1}} \varphi_{1}\left(2^{j}(y-x)\right)(y+x)^{-1 / q^{\prime}} d y\right|^{p}|x|^{a} d x \\
& \geq c \int_{I_{-j-2}} 2^{\frac{j p}{q}+\frac{j p}{q}-a}\left|\int_{I_{1}} \varphi_{1}\left(2^{j}(y-x)\right) d y\right|^{p} d x \\
& \geq\left. c \int_{I_{-j-2}} 2^{\frac{j p}{q}+\frac{j p}{q}-a}||_{\left\{y: 0<2^{j}(x-y)-\frac{1}{2} 2^{j}\right\}} \varphi_{1}\left(2^{j}(y-x)\right) d y\right|^{p} d x \\
& \geq c \int_{I_{-j-2}} 2^{\frac{j p}{q}+\frac{j p}{q}-a-\frac{j p}{2 q}} d x=c 2^{j\left(p-a-\frac{p}{2 q}-1\right)}=c 2^{j \frac{p}{p_{3}}-a-1} .
\end{aligned}
$$

The fourth inequality follows since it is easy to check that, for $x \in I_{-j-2}$, $\left\{y: 0<2^{j}(x-y)-\frac{1}{2}<2^{j}\right\} \subset I_{1}$. Now, for $2^{j \frac{p}{p_{3}}-a-1}$ to be bounded on $j<0$, it must happen that $\frac{p}{p_{3}}-a-1>0$.

\section{ACKNOWLEDGEMENT}

The author was partially supported by Agencia Córdoba Ciencia, Secyt-UNC and Conicet.

\section{REFERENCES}

1. R. R. Coifman and C. Fefferman, Weighted norm inequalities for maximal functions and singular integrals. Studia Math. 51(1974), 241-250.

2. J. Duonndikoetxea, Fourier analysis. (Translated from the Spanish) Graduate Studies in Mathematics, 29. American Mathematical Society, Providence, RI, 2001; Spanish original: Análisis de Fourier. Ediciones de la Universidad Autónoma de Madrid, Editorial Siglo XXI, 1990.

3. J. Duonndikoetxea and J. L. Rubio de Francia, Maximal and singular integral operators via Fourier transform estimates. Invent. Math. 84(1986), No. 3, 541-561.

4. T. Godoy and M. Urciuolo, About the $L^{p}$-boundedness of some integral operators. Rev. Un. Mat. Argentina 38(1993), No. 3-4, 192-195.

5. T. Godoy and M. Urciuolo, On certain integral operators of fractional type. Acta Math. Hungar. 82(1999), No. 1-2, 99-105.

6. B. Muckenhoupt, Weighted norm inequalities for the Hardy maximal function. Trans. Amer. Math. Soc. 165(1972), 207-226. 
7. F. Ricci and P. SJÖGREn, Two-parameter maximal functions in the Heisenberg group. Math. Z. 199(1988), No. 4, 565-575.

8. M. S. Riveros and M. Urciuolo, Weighted inequalities for integral operators with some homogeneous kernels. Czechoslovak Math. J. 55(130)(2005), No. 2, 423-432.

9. D. K. WAtson, Vector-valued inequalities, factorization, and extrapolation for a family of rough operators. J. Funct. Anal. 121(1994), No. 2, 389-415.

(Received 10.03.2005)

Author's address:

Famaf-Ciem, Universidad Nacional de Córdoba

Medina Allende s/n, Ciudad Universitaria

5000 Córdoba

Argentina

E-mail: urciuolo@mate.uncor.edu 